\title{
Uso inconsistente del condón entre trabajadoras sexuales en Ecuador: resultados de una encuesta de comportamientos
}

\author{
Juan Pablo Gutiérrez, M en Econ de la Sal, ${ }^{(1)}$ D iana Molina-Yépez, M Esp en Inves y Adm en Sal, ${ }^{(2)}$
} Fiona Samuels, Dra en Antr Soc, ${ }^{(3)}$ Stefano Michele Bertozzi, Dr en Ger y Pol de Sal. ${ }^{(1,4)}$

\section{Gutiérrez JP, Molina-Yépez D, Samuels F, Bertozzi SM. Uso inconsistente del condón entre trabajadoras sexuales en Ecuador: resultados de una encuesta de comportamientos. Salud Publica Mex 2006;48:104-112.}

\section{Resumen}

Objetivo. 0 btener información sobre la tasa de uso del condón y otros comportamientos y características relacionados con el riesgo de adquirir infecciones de transmisión sexual en trabajadoras sexuales (TS) de ocho ciudades del Ecuador. Este estudio presenta resultados de una encuesta realizada conTS en ocho ciudades del Ecuador. Material y métodos. Se trata de un estudio transversal en el que se aplicó un cuestionario de comportamientos, actitudes y características socioeconómicas y demográficas a una muestra deTS en las ocho ciudades del Ecuador que concentran a la mayor parte de la población del país y padecen la mayor problemática en relación con el VIH. Resultados Se obtuvo información de un total de $2867 \mathrm{TS}$, la mayor parte de ellas en sus lugares de trabajo. El mayor porcentaje de lasTS entrevistadas realizaba sus actividades en sitios dedicados al comercio sexual. La edad media de las entrevistadas fue de 28 años (IC95\% 27-29) y alrededor de la mitad vivía con parejas masculinas (ya sea casadas 0 en unión libre). La tasa de uso del condón con el último cliente fue de $88 \%$ ( $82 \%$ de forma consistente); en cambio, con las parejas regulares fue de 6\%. Un alto índice de habilidades para la vida, mayor nivel socioeconómico y contar con permiso para trabajar
\end{abstract}

\author{
Gutiérrez JP, Molina-Yépez D, Samuels F, Bertozzi SM. \\ Inconsistent condom use among sexual \\ workers in Ecuador: results \\ from a behavior survey. \\ Salud Publica Mex 2006;48:104-112.
}

\begin{abstract}
A bstract
Objective. W hilst existing data suggests that the HIV epidemic in Ecuador is concentrated amongst men who have sex with men (MSM), there is very little available information on the situation of key populations, i.e. those most at risk of HIV infection and/or transmitting the infection. In particular, there is very little known about sex workers (SW s), their rate of condom use and other behaviors and characteristics with respect to the risk of acquiring sexually transmitted infections (STIS). This study presents findings from a survey carried out with SW $s$ in eight cities in Ecuador. Material and Methods. U sing a cross-sectional design, a questionnaire focusing on behaviours, attitudes and socio-economic and demographic characteristics was administered to SW s in eight cities in Ecuador. These eight cities together account for the majority of the population in the country, and they were also identified as the locations with high reported levels of HIV. Results. Information from a total of 2867 SW s was obtained, the majority were captured in their workplaces. Most of SW s inter viewed carry out their activities in closed settings dedicated to sex work (i.e. not in the street). The average age of respondents was $28(95 \% \mathrm{Cl} 27-29)$, and around half of them live with a
\end{abstract}

Este estudio forma parte del Proyecto Prevención en las Fronteras, que conduce la Alianza Internacional contra el VIH/SIDA, a través de la Corporación Kimirina en Ecuador, y ha sido financiado por la Fundación Bill y Melinda Gates.

(1) División de Economía y Políticas de la Salud, Centro de Investigación en Sistemas de Salud, Instituto N acional de Salud Pública. Cuernavaca, Morelos, México.

(2) Instituto Juan César García. Q uito, Ecuador.

(3) Unidad de Investigación y Evaluación, A lianza Internacional contra el VIH/SIDA. Brighton, Reino Unido.

(4) División de Economía, Centro de Investigación y Docencia Económicas, A.C. (CID E). México, D.F., México.

Fecha de recibido: 22 de abril de 2005 - Fecha de aprobado: 6 de diciembre de 2005

Solicitud de sobretiros: Juan Pablo Gutiérrez, D ivisión de Economía y Políticas de la Salud, Instituto N acional de Salud Pública,Av. Universidad 655, colonia Sta. María A huacatitlán, 62508 Cuernavaca, Morelos, México.

Correo electrónico: jpgutier@ correo.insp.mx 
se relacionaron de forma positiva con el uso del condón con clientes (RP=1.40; IC95\% 1.40-1.40; RP= 1.37; IC95\% 1.36-1.37; y $\mathrm{RP}=7.26$; IC95\% 6.87-7.46, respectivamente). Conclusiones Si bien el uso del condón de las TS ecuatorianas con clientes es elevado, disminuye si se analiza su consistencia y es sumamente bajo cuando se trata de parejas regulares. La utilización del condón, por otra parte, parece relacionarse con variables sobre las cuales es posible realizar intervenciones para modificarlas, como las habilidades para la vida y el permiso oficial para realizar el trabajo sexual. En ese sentido, es importante realizar intervenciones específicas que incrementen el uso consistente del condón en esta población.

Palabras clave: trabajo sexual; prevención; enfermedades sexualmente transmisibles; VIH; Ecuador male partner (married or not).The rate of condom use with the last client was $88 \%$ ( $82 \%$ consistently with the last three), whilst with regular partners it was $6 \%$. A high index of lifeskills, high socio-economic status and having an official document that allows them to work, were positively associated with condom use with clients (PR [Cl 95\%] 1.40 [1.40-1.40], 1.37 [1.36-1.37], y 7.26 [6.87-7.46], respectively). Conclusions.W hilst condom use with clients amongst Ecuadorian SW $\mathrm{s}$ is high, this diminishes if one analyzes consistent condom use and is notably low with respect to regular partners. Condom use appears to be related to variables that can be linked to interventions, e.g. life-skills and official permission to carry out sex work. It is, therefore, important to tailor interventions for this population so they maximize the likelihood to increase consistent condom use.

Key words: sex work; prevention; sexually transmitted infections; HIV; Ecuador a epidemia del VIH/SIDA en Ecuador, al igual que en la mayoría de los países latinoamericanos, se caracteriza por concentrarse en grupos que tienen prácticas de riesgo. Si bien la prevalencia general en adultos es de sólo $0.3 \%$, se han notificado prevalencias entre grupos HSH de hasta $16 \%$, ${ }^{1}$ en tanto que entre las trabajadoras sexuales (TS) se han publicado datos de $0.5 \%$ a $1 \% .^{2}$ De esta forma, la prevención de la transmisión entre las poblaciones clave para la epidemia es de particular importancia para enfrentarla.

La falta de información sobre los comportamientos sexuales ha hecho difícil explicar por qué las tasas de infección han permanecido relativamente bajas entre las trabajadoras sexuales (TS), mientras que son elevadas entre los $\mathrm{HSH}^{2-4}$ En cambio, se sabe que la principal vía de transmisión es la sexual, lo que obliga a identificar los riesgos de las TS que podrían exponerlas a una epidemia mayor, en virtud del elevado número de contactos sexuales que tienen, algo que se ha señalado desde $1994 .^{5}$

Una búsqueda por "trabajo sexual", "prostitución" y "Ecuador" en la base más consultada en biomedicina, la de la National Library of Medicine de Estados Unidos, ${ }^{6}$ presenta sólo una publicación con resultados regionales sobre epidemiología molecular del VIH-1 en cinco países sudamericanos. ${ }^{2}$ Incluso si se extiende la revisión a Sudamérica, las publicaciones halladas corresponden a otros países de la región y ninguna a Ecuador. De forma similar, en la base del AIDS Education Global Information System (AEGIS), ${ }^{7}$ que incluye publicaciones en prensa, informes y resúmenes en conferencias internacionales, se identifican 19 publicaciones sobre prostitución y Ecuador, de las cuales cinco son notas en medios masivos con referencia a la situación del VIH en Ecuador. De los 14 restantes, dos se refieren a trabajadoras sexuales ecuatorianas en España y dos a contactos de trabajadoras sexuales con marinos en Chile. Otras dos describen el proyecto del que trata este documento. Cuatro más tratan sobre programas de prevención o intervenciones dirigidas a las TS. Por último, el grupo restante de trabajos se presentó en las Conferencias Internacionales de SIDA en 1989 y 1992, en las que se notificaron resultados de encuestas serológicas para estimar la prevalencia de VIH en el Ecuador. La principal conclusión de estos estudios fue la baja prevalencia en la población general y la mayor prevalencia entre HSH, así como un incremento observado de las prevalencias con el tiempo. ${ }^{8-11}$

Si bien no se han publicado tasas elevadas de infección por VIH entre las TS en la región latinoamericana, hay evidencias que sugieren altas prevalencias de otras infecciones de transmisión sexual, en particular el virus del herpes simple tipo $2 .{ }^{12-15}$ Estudios efectuados en Perú, país fronterizo con Ecuador, comunican también tasas aumentadas de otras ITS, como Chlamydia y gonorrea. ${ }^{16}$

El conocimiento insuficiente de las características de las TS se ha convertido en un obstáculo para el desarrollo de conductas de prevención efectivas. Cuando existe información, su calidad es limitada. Es importante entender de forma más precisa los mecanismos que intervienen en el uso del condón en esta población, las barreras para negociarlo y en general la estructura del mercado del comercio sexual, además de la negociación que es factible con sus parejas estables, para delinear de esta forma intervenciones 
preventivas que se ajusten a su marco de referencia. El énfasis en comportamientos como una oportunidad de intervención para prevenir infecciones es la base del concepto de vigilancia epidemiológica de segunda generación, que promueve ONUSIDA. ${ }^{17}$

El éxito de las intervenciones de prevención dirigidas a las TS para incrementar el empleo del condón con clientes en países africanos y asiáticos ha mostrado que es posible realizar intervenciones efectivas con TS para reducir las prácticas de riesgo. ${ }^{18-20}$ No obstante, el reto para los países latinoamericanos es en cierta forma distinto: si bien se ha logrado desde hace años un elevado uso del condón con los clientes, éste no es consistente, y es prácticamente nulo con las parejas regulares. ${ }^{21}$

En este contexto, el diseño de intervenciones debe buscar incrementar la utilización consistente del preservativo, así como desarrollar medidas para promover su uso con parejas regulares.

Esta investigación muestra un panorama de las prácticas de riesgo de las TS en el Ecuador y diferencia lo que ocurre con los clientes y con las parejas regulares. Asimismo, ofrece un análisis de las características de las TS que se vinculan con un mayor uso consistente del condón. Este estudio forma parte de la evaluación del Proyecto Prevención en las Fronteras (PPF), una iniciativa de prevención de VIH basada en la focalización de intervenciones de prevención dirigidas a poblaciones clave en áreas geográficas e ideadas con la participación activa de la población objetivo.

\section{Material y métodos}

\section{Metodología}

Como parte de las actividades de evaluación del efecto del proyecto PPF, se llevó a cabo una encuesta basal con HSH y TS en las ocho ciudades de Ecuador seleccionadas para el proyecto. La agencia encargada del proyecto de prevención, a través de un análisis situacional, identificó de forma previa a dichas ciudades con base en su mayor problemática en relación con el $\mathrm{VIH} /$ SIDA.

El objetivo de la línea basal fue establecer la situación antes de la intervención de las trabajadoras sexuales respecto de los comportamientos de riesgo, características demográficas y socioeconómicas, así como actitudes y conocimientos sobre VIH e ITS. Esta información se recogió con dos propósitos: informar el diseño de las intervenciones y servir de punto de referencia para la valoración del efecto del programa en un momento posterior. A continuación se describe la metodología del estudio que es relevante para el análisis presentado en este documento.
La Comisión de Ética del Instituto Nacional de Salud Pública de México, el Comité de Ética del Consejo Nacional en el Ecuador y la Alianza Internacional de VIH/SIDA concedieron la aprobación a este protocolo.

\section{Muestra}

Se calculó el tamaño de la muestra para medir los cambios observados en el uso del condón entre la línea basal y las encuestas de seguimiento, tras considerar el efecto de diseño de la selección aleatoria de las ciudades. El uso del condón con la última pareja se tomó como una variable dicotómica, con una diferencia observable en el seguimiento de 10 puntos porcentuales y un efecto de diseño de 2.2.

Como se indicó, las ciudades se preseleccionaron para el estudio. Con la finalidad de calcular el tamaño de la población de estudio en cada ciudad se trazó un mapeo en cada una de las ciudades con la participación de las mismas poblaciones objetivo. Con base en los resultados de este mapeo y las dimensiones del tamaño total de la muestra se asignó un tamaño por localidad, que en la mayoría de los casos abarcaba a la totalidad de las TS visibles; por esa razón, al realizar la encuesta se intentó entrevistar, en los puntos visitados, a todas las TS presentes.

\section{Recolección de datos}

El instrumento para recolectar la información lo desarrolló un equipo internacional multidisciplinario e interinstitucional, que coordinó el investigador encargado de la evaluación del PPF, con la participación de los investigadores locales y otros actores clave.

El cuestionario incluía: información sobre variables sociodemográficas (edad, educación, situación laboral, bienes); datos sobre comportamientos sexuales, en particular información sobre el tipo de parejas sexual o cliente, prácticas sexuales, empleo del condón, tarifa cobrada y otros detalles sobre los tres clientes más recientes; información sobre la pareja regular (en caso de que existiera), definida como pareja estable no comercial; y datos sobre conocimientos de VIH/SIDA e ITS en general y actitudes hacia personas con VIH/SIDA.

Para ajustar los cuestionarios al lenguaje apropiado de las TS en Ecuador y depurar su contenido, se llevaron a cabo una serie de grupos focales y entrevistas con trabajadoras sexuales en la ciudad de Quito. Asimismo, se realizaron dos pruebas piloto en ciudades distintas a las del estudio y sus resultados se utilizaron para adecuar los cuestionarios; los ajustes realizados fueron de forma (lenguaje). 
Antes de la segunda prueba piloto se capacitó a los entrevistadores; se trató de establecer un enfoque estandarizado de entrevistas y destacar los principios fundamentales de las técnicas de la entrevista. Los entrevistadores y supervisores revisaron el cuestionario con los investigadores para homologar los contenidos y el proceso adecuado para la entrevista. En virtud de la sensibilidad de los temas y las particularidades de las poblaciones, se decidió invitar como entrevistadores(as) a integrantes de las poblaciones; esto facilitó el acceso a los sitios de trabajo de las TS y el establecimiento de rapport.

Asimismo, los entrevistadores de las poblaciones clave habían participado antes en el mapeo, por lo que tenían un adecuado conocimiento de las ciudades y los puntos de trabajo de las TS.

La recolección de información se llevó a cabo entre julio y septiembre de 2003 mediante entrevistas confidenciales con las TS, la mayoría de ellas en los sitios de trabajo y otra parte en las clínicas que prestan servicio a esta población. En los sitios de trabajo, las TS que aceptaron participar contestaron el cuestionario en el tiempo que esperaban clientes. Dado que no se registraron los nombres de las TS, no es posible relacionar los datos con personas específicas.

Antes de cada entrevista se les explicó a los participantes el objetivo del estudio y la participación requerida de ellos y se obtuvo su consentimiento informado y firmado para participar en el estudio. Una copia del consentimiento se entregó a las TS y en ella se incluía la información de contacto con los investigadores.

Las entrevistas se efectuaron en los lugares de trabajo de las TS o en centros de salud en los horarios y espacios de atención a ellas; se invitó a participar a las mujeres que se encontraban en estos lugares y se les explicó el contenido de la entrevista.

Para garantizar la calidad de las entrevistas y el apego a los procedimientos éticos, por cada cinco entrevistadores un supervisor verificaba la aplicación correcta de las entrevistas.

\section{A nálisis estadístico}

La digitalización de los datos se llevó a cabo mediante LSD (Sistemas Integrales, Santiago de Chile) y los datos se analizaron con el programa Stata 8.0. Para garantizar que los datos de la base de datos correspondían a los registros de los cuestionarios, se capturó dos veces cada cuestionario y se corrigieron las inconsistencias. Se revisaron las variables incluidas en este análisis para eliminar los valores aberrantes.

La base de datos se modificó para generar un panel con los clientes, en el que se contaba con tres observaciones por cada TS. De esta forma, se definió como evento cada una de las transacciones y se reordenó la base para contar con 8601 eventos (tres por cada TS). Tanto para las estadísticas descriptivas como para las regresiones se consideró el efecto de diseño para calcular los errores. Para todos los análisis se asumió un nivel de significancia de $95 \%$.

Se generaron en primera instancia estadísticas descriptivas de las características sociodemográficas de las TS y la prevalencia de los comportamientos de riesgo. Para estimar la correlación entre comportamientos de riesgo y variables sociodemográficas se ajustó un modelo de regresión logística, con el uso del condón con los clientes como variable dependiente con base en el panel de clientes construido. Para incluir el hecho de que las observaciones estaban agrupadas de dos formas (por TS y ciudad), se estimó un modelo que introdujo efectos fijos por ciudad y efectos aleatorios por TS.

Para los modelos de regresión, se generaron variables categóricas para la edad, el tiempo en el comercio sexual, los años de escolaridad y el precio pagado por los condones. En todos los casos, las categorías se crearon a partir de la distribución observada de la variable y se obtuvieron tres o cuatro grupos.

De manera adicional, se generaron tres índices que se incluyeron en los modelos: nivel socioeconómico, capital social y habilidades para la vida.

El índice de nivel socioeconómico se creó con el ingreso neto, la propiedad de bienes y el nivel educativo y se utilizó el método de componentes principales. El puntaje obtenido se categorizó en cuartiles.

El indicador de capital social resultó del promedio de las respuestas relacionadas con la disposición de otra persona que las apoyara en diferentes actividades (recurrir a alguien cuando se requiere dinero o se busca atención médica, conversar los problemas, necesidad de comida o alojamiento y, en caso de violencia, abusos de clientes o clientes violentos).

Por su parte, el índice de habilidades para la vida se construyó con el promedio de respuestas afirmativas a los siguientes factores: tener control sobre su vida, lograr que los clientes utilicen el preservativo, tener acceso a servicios de VIH/SIDA, tomar la iniciativa para usar el condón, influir en las decisiones del trabajo, rechazar a un cliente que se rehúsa a usar el condón o, si se siente amenazada, trabajar entre TS de manera conjunta, dado que la organización de las TS ayuda a incrementar de empleo del preservativo.

Para los índices de capital social y habilidades para la vida se adoptó la metodología utilizada en un estudio similar efectuado en la India. ${ }^{22}$

Debido a que se ha aducido que al interpretar las razones de momios $(R M)$ es posible sobreestimar el riesgo (o protección) que se atribuye a alguna varia- 
ble, ${ }^{23}$ además de las $R M$ que resultan de la regresión logística, se calcularon las razones de prevalencia RP correspondientes de acuerdo con la fórmula sugerida por Zhang y Yu. ${ }^{24}$

\section{Resultados}

\section{Características sociodemográficas}

Se recolectó información de 2867 trabajadoras sexuales en las ocho ciudades. Del total, $80 \%$ se entrevistó en el sitio de trabajo y el restante $20 \%$ en clínicas o centros de salud. En el primer caso, poco más de la mitad trabajaba en "boliches", espacios cerrados amplios en los que hay varios cuartos (pueden ser más de 100) y en los que también se expenden bebidas alcohólicas. Sólo $11 \%$ del total se entrevistó en la calle (cuadro I).

Como se puede observar en el cuadro II, la edad promedio de las TS participantes fue de 28 años (IC95\% 27.1-28.8) y poco más de 54\% señaló que vivía con alguna pareja masculina; las demás informaron que eran solteras. La inmensa mayoría (86\%) refirió tener al menos un hijo, con un promedio de dos.

Si bien la mayor parte de las TS residía en la ciudad en la que se entrevistaron, 38\% dijo que sólo iba a trabajar a las ciudades.

Por otra parte, 91\% sabía leer y podía escribir un recado y el promedio de años de escuela fue de 7.3 años (IC95\% 6.8-7.7). Tan sólo 15\% tenía otra ocupación, además del trabajo sexual; este porcentaje es similar al de las que dijeron no considerarse trabajadoras sexuales, si bien llevaron a cabo al menos una transacción de sexo por dinero.

\section{Cuadro I}

\section{Distribución de TS POR LUGAR DE ENTREVISTA}

\begin{tabular}{lrc} 
Lugar & Número (porcentaje) \\
Bar o cervecería & 25 & $(1)$ \\
\hline Boliche o casa de citas & 1119 & $(43)$ \\
\hline Calle & 308 & $(12)$ \\
\hline Centro de salud & 539 & $(19)$ \\
\hline Sitios públicos & 19 & $(<1)$ \\
\hline Hotel & 91 & $(3)$ \\
\hline Club & 396 & $(14)$ \\
\hline ON G & 10 & $(<1)$ \\
\hline Otros & 360 & $(13)$ \\
\hline Total & 2867 &
\end{tabular}

Encuesta basal, evaluación del Proyecto Prevención en las Fronteras, Ecuador, 2003

\section{Cuadro II \\ Características de las TS participantes}

Característica

Media o porcentaje*

Edad

27.9

$(27.1-28.8)$

\begin{tabular}{lc}
\hline Viven con pareja masculina & $54 \%$ \\
\hline Solteras & $46 \%$ \\
\hline Tienen al menos un hijo & $86 \%$ \\
\hline Número de hijos promedio de las que tienen hijos & 2 \\
\hline Viven en la ciudad en la que trabajan & $62 \%$ \\
\hline Saben leer y escribir un recado / carta & $91 \%$ \\
\hline Años de escolaridad & 7.3
\end{tabular}

Años de escolaridad

$(6.8-7.7)$

\begin{tabular}{lc}
\hline Tienen un trabajo adicional & $15 \%$ \\
\hline Se consideran trabajadoras sexuales & $85 \%$ \\
\hline Trabajan todas las semanas del mes & $52 \%$ \\
\hline Semanas trabajadas para las que no trabajaron el mes & 2.1 \\
& $(2.0-2.3)$ \\
\hline N úmero de horas trabajadas la semana anterior & 39 \\
& $(34-44)$ \\
\hline N úmero de clientes la semana anterior & 22 \\
\end{tabular}

Número de clientes rechazados la semana anterior

(3-4)

Años en comercio sexual

5.2

(4.4-6.1)

\begin{tabular}{ll}
\hline Tienen documento de trabajo & $82 \%$ \\
\hline Tienen pareja regular & $58 \%$
\end{tabular}

Número de veces que tuvieron relaciones

con su pareja regular

3.5

(3.3-3.7)

Tuvieron sexo con penetración la última ocasión

\begin{tabular}{lc} 
Con su pareja regular & $86 \%$ \\
\hline Usaron condón en la última relación con penetración & \\
con la pareja regular & $6 \%$ \\
\hline Uso del condón con el último cliente & $88 \%$ \\
\hline Uso consistente del condón con clientes & $82 \%$
\end{tabular}

* En el caso de las variables continuas se registran la media y el intervalo de confianza de $95 \%$ para la estimación, que considera el efecto de diseño. Para las variables categóricas se registra el porcentaje

Encuesta basal, evaluación del Proyecto Prevención en las Fronteras, Ecuador, 2003

\section{Parejas regulares}

Por lo que se refiere a las parejas sexuales regulares, $58 \%$ de las TS tenía una al momento de la encuesta, con la que sostenía relaciones sexuales en promedio 3.5 veces durante la semana anterior a la encuesta. 
En cuanto a la última ocasión, se practicó la penetración vaginal o anal en $86 \%$ de las veces y de ellas sólo en $6 \%$ de las veces se utilizó el condón.

\section{Características del trabajo sexual}

Por lo que se refiere al trabajo sexual, $52 \%$ de las TS había trabajado durante todas las semanas del mes anterior, en tanto que el resto lo hizo dos semanas en promedio. De las que habían trabajado durante la semana anterior a la encuesta, laboraron 40 horas en promedio en la semana y tuvieron alrededor de 21 clientes, es decir, si bien el tiempo efectivo con cada cliente es de alrededor de cinco minutos, dedican casi dos horas por cliente para el proceso de búsqueda y negociación. Las TS rechazaron en promedio a cuatro clientes por diversas razones, lo que representa alrededor de $20 \%$ de los clientes efectivos.

De acuerdo con lo que señalaron, las TS participantes tenían en promedio poco más de cinco años en el comercio sexual, aunque la media de años fue de tres, con un límite intercuartil de 2-7 años (25 y 75\%). Alrededor de $82 \%$ de las TS contaba con el documento oficial de registro que le permitía trabajar de forma legal; resulta de interés que el porcentaje se reduce con los años de actividad en el comercio sexual: $76 \%$ para las que refirieron más de siete años de experiencia laboral. Es pertinente considerar que las TS pierden el documento casi siempre por no someterse a las pruebas de ITS o resultar positivas en ellas.

\section{Uso del condón con clientes}

Si bien el uso observado del condón con el cliente más reciente fue elevado (87\%), también se reconoció una notoria variabilidad entre una ciudad y otra; así, mientras en una el empleo notificado llegó hasta $97 \%$, en la ciudad con el nivel más bajo se registró sólo $70 \%$.

Con base en la información recogida de los tres clientes más recientes, se estimó una medida de uso consistente del preservativo, definida como el empleo en los tres clientes últimos, y una media de uso inconsistente, determinada como el uso de al menos uno. A partir de esta medida, el porcentaje de TS que informaron utilización consistente fue de $82 \%$ y los límites entre ciudades fluctuó entre 65 y 96\%.

\section{Determinantes de uso del condón con parejas regulares}

Por lo que respecta al empleo del condón con parejas regulares, se analizó el posible nexo con variables demográficas y socioeconómicas, así como los indicado- res creados de capital social y habilidades para la vida. $\mathrm{Ni}$ de forma bivariada ni dentro de un modelo multivariado se identifica alguna vinculación. La única variable que se correlacionó con la utilización del preservativo con una pareja regular fue el uso consistente del condón con clientes, con una razón de prevalencias de 2.47 (IC95\% 1.03-4.56), como se muestra en el cuadro III.

\section{Determinantes de uso del condón con clientes}

Como se señaló en la sección de métodos, para analizar el empleo del preservativo con los clientes se aprovechó la existencia de tres observaciones por TS con objeto de crear un panel que hiciera posible analizar los determinantes de uso del condón con los clientes con una muestra de sucesos mayor.

Con este panel se ajustó un modelo de regresión logística en el que se incluyeron como variables explicativas aquellas para las que existía apoyo teórico como probables factores que podrían afectar de manera positiva o negativa la utilización del condón. Los resultados de este modelo se presentan en el cuadro IV.

Los años de educación se incluyeron en el modelo para observar si había algún efecto adicional por educación que no se relacionara con el nivel socioeconómico. Los resultados sugieren que la conclusión de los estudios primarios se vincula de modo positivo con el uso del condón, al margen del nivel socioeconómico, lo que podría resaltar un efecto adicional por educación.

Respecto de la edad de las TS, se advierte una notoria relación para todos los grupos de edad en comparación con las más jóvenes: el uso del preservativo disminuye con la edad de forma progresiva y consistente. Este resultado sugiere diferencias generacionales quizá vinculadas con la utilización del dispositivo, es decir, es posible que las TS iniciadas en la actividad

\section{Cuadro III \\ Razón de Momios (IC 95\%) PARA uso del CONDón DE LASTS CON PAREJA REGULAR EN FUNCIÓN DEL USO CONSISTENTE DEL CONDÓN CON CLIENTES}
Variable
$\mathrm{RM} *$
$R P^{\ddagger}$
U so consistente del condón con clientes

3.18
$(1.03-9.82)$
2.47
$(1.03-4.56)$
* El intervalo de confianza de $95 \%$ considera el efecto de diseño
₹ Calculada con la fórmula RP= RM /[(1-P $\left.)+\left(P_{0} * R M\right)\right]$ de Zhang J,Yu K ${ }^{24}$

Encuesta basal, evaluación del Proyecto Prevención en las Fronteras, Ecuador, 2003 


\section{Cuadro IV}

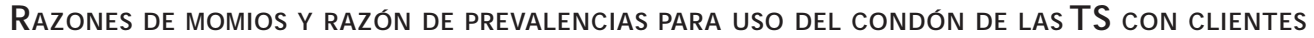

\begin{tabular}{|c|c|c|c|}
\hline Variable & Categorías & RM* (IC95\%) & RP equivalente (IC95\%) \\
\hline Vive con pareja hombre & No & 1.0 & 1.0 \\
\hline & Sí & $\begin{array}{c}1.48 \\
(0.91-2.43)\end{array}$ & $\begin{array}{c}1.22 \\
(0.95-1.48)\end{array}$ \\
\hline \multirow[t]{3}{*}{ Años de escolaridad } & Hasta 5 años & 1.0 & \\
\hline & 6 a 8 años & $\begin{array}{c}2.29 \\
(1.17-4.46)\end{array}$ & $\begin{array}{c}1.3 \\
(1.06-1.47)\end{array}$ \\
\hline & 9 años o más & $\begin{array}{c}1.51 \\
(0.74-3.07)\end{array}$ & $\begin{array}{c}1.16 \\
(0.87-1.38)\end{array}$ \\
\hline \multirow[t]{4}{*}{ Edad } & 13 a 21 años & 1.0 & \\
\hline & 22 a 25 años & $\begin{array}{c}0.19 \\
(0.06-0.63)\end{array}$ & $\begin{array}{c}0.47 \\
(0.19-0.86)\end{array}$ \\
\hline & 26 a 32 años & $\begin{array}{c}0.07 \\
(0.02-0.23)\end{array}$ & $\begin{array}{c}0.21 \\
(0.07-0.52)\end{array}$ \\
\hline & 33 años y más & $\begin{array}{c}0.01 \\
(0.0-0.02)\end{array}$ & $\begin{array}{c}0.04 \\
(0-0.08)\end{array}$ \\
\hline \multirow{4}{*}{ Años como TS } & Hasta 1 año & 1.0 & \\
\hline & 2 años & $\begin{array}{c}0.22 \\
(0.10-0.47)\end{array}$ & $\begin{array}{c}0.66 \\
(0.43-0.86)\end{array}$ \\
\hline & 3 a 6 años & $\begin{array}{c}0.31 \\
(0.15-0.63)\end{array}$ & $\begin{array}{c}0.59 \\
(0.3-0.84)\end{array}$ \\
\hline & 7 años y más & $\begin{array}{c}0.10 \\
(0.05-0.23)\end{array}$ & $\begin{array}{c}0.38 \\
(0.22-0.62)\end{array}$ \\
\hline \multirow[t]{3}{*}{ Precio del condón } & Bajo & 1.0 & \\
\hline & Medio & $\begin{array}{c}0.02 \\
(0.01-0.05)\end{array}$ & $\begin{array}{c}0.06 \\
(0.03-0.15)\end{array}$ \\
\hline & Alto & $\begin{array}{c}0.01 \\
(0.01-0.05)\end{array}$ & $\begin{array}{c}0.03 \\
(0.03-0.15)\end{array}$ \\
\hline \multirow[t]{3}{*}{ Habilidades para la vida } & Bajo & 1.0 & \\
\hline & Medio & $\begin{array}{c}156.01 \\
(54.3-447.51)\end{array}$ & $\begin{array}{c}1.6 \\
(1.59-1.6)\end{array}$ \\
\hline & Alto & $\begin{array}{c}124.63 \\
(53.60-289.76)\end{array}$ & $\begin{array}{c}1.4 \\
(1.39-1.4)\end{array}$ \\
\hline \multirow[t]{3}{*}{ Capital social } & Bajo & 1.0 & \\
\hline & Medio & $\begin{array}{c}0.64 \\
(0.31-1.32)\end{array}$ & $\begin{array}{c}0.84 \\
(0.57-1.09)\end{array}$ \\
\hline & Alto & $\begin{array}{c}0.18 \\
(0.09-0.34)\end{array}$ & $\begin{array}{c}0.42 \\
(0.25-0.63)\end{array}$ \\
\hline \multirow[t]{4}{*}{ N ivel socioeconómico } & Bajo & 1.0 & \\
\hline & Medio bajo & $\begin{array}{c}3.39 \\
(1.63-7.08)\end{array}$ & $\begin{array}{c}1.22 \\
(1.11-1.28)\end{array}$ \\
\hline & Medio alto & $\begin{array}{c}1.55 \\
(0.79-3.04)\end{array}$ & $\begin{array}{c}1.1 \\
(0.94-1.2)\end{array}$ \\
\hline & Alto & $\begin{array}{c}16.11 \\
(6.08-42.68)\end{array}$ & $\begin{array}{c}1.35 \\
(1.3-1.36)\end{array}$ \\
\hline \multirow[t]{2}{*}{ Tienen permiso para trabajar } & No & 1.0 & \\
\hline & Sí & $\begin{array}{c}125.03 \\
(58.47-267.38)\end{array}$ & $\begin{array}{c}7.26 \\
(6.87-7.47)\end{array}$ \\
\hline
\end{tabular}

* El intervalo de confianza de $95 \%$ considera el efecto de diseño con efectos fijos por ciudad, así como la correlación intraindividual, con un modelo de efectos aleatorios individuales

₹ Calculada con la fórmula RP= RM $/\left[\left(1-P_{0}\right)+\left(P_{0} * R M\right)\right]$ de Zhang $], Y u K^{24}$

Encuesta basal, evaluación del Proyecto Prevención en las Fronteras, Ecuador, 2003 
antes de que el uso del condón se promoviera de forma general son justamente las que lo emplean menos.

Este hallazgo se apoya en el nexo observado con la experiencia en el trabajo sexual, que en la misma dirección sugiere que aquellas que tienen más tiempo dedicadas a esta actividad son más proclives a prescindir del preservativo con los clientes.

Por lo que se refiere a los indicadores de habilidades para la vida y el capital social, se detectaron relaciones sólidas, positivas para el primero y negativas para el segundo. No es claro el mecanismo para la correlación negativa de la variable de capital social y obliga a explorar de manera más amplia este punto. Por otra parte, la obtención de un valor más alto de habilidades para la vida resulta un poderoso predictor de uso del condón, lo que revela un efecto de dosis considerable.

De igual manera, el indicador socioeconómico se correlacionó sin duda con el empleo del preservativo, si bien el efecto de dosis no es del todo claro. No obstante, es evidente que las TS con un mejor nivel económico son asimismo las que notificaron un mayor uso del dispositivo.

En concordancia con este resultado, el precio de los condones según los datos informados se vinculó en grado positivo con la utilización del condón y se identificó un efecto de dosis (la prevalencia de uso del condón se reduce con el incremento de su precio).

Por último, tener un documento oficial para trabajar posee un nexo innegable con el empleo del preservativo. La razón de prevalencias para el uso del condón que se obtuvo en esta investigación resultó la más importante en el análisis multivariado.

\section{Discusión}

Los resultados presentados en este artículo son los primeros datos publicados sobre una muestra grande de trabajadoras sexuales en el Ecuador. Estos datos señalan que si bien, al igual que en otros países de la región, las TS señalan un elevado uso del condón con los clientes, éste no es consistente; además, existen notables diferencias con otras ciudades, lo cual debe tomarse en cuenta en la planeación de las medidas de prevención de ITS dirigidas a esta población.

Asimismo, resalta la presencia de un conjunto de factores que se correlacionan de forma notoria con el uso del condón y la mayoría de ellos es susceptible de intervención. Si bien el nivel socioeconómico, uno de los factores encontrados, no es una variable en la que los programas de salud puedan incidir de manera directa, podría subrayar la importancia de las potenciales sinergias entre los programas de salud y los enfocados en mejorar las condiciones de vida de las poblaciones. En años recientes se ha propuesto que las políticas de combate a la pobreza son en realidad una forma de prevención de infecciones como el VIH y otras ITS. ${ }^{25}$

Por otra parte, la relevancia que mostró el indicador de habilidades para la vida y la disposición de un documento oficial para trabajar, que fueron las otras dos variables sólidamente relacionadas con el empleo de condón, pueden ser objetivos de intervenciones desde los programas de salud pública.

Las habilidades para la vida, como se definieron para este análisis, se refieren sobre todo a la percepción de las TS de poder incidir en la utilización del preservativo, lo que en esencia se vincula con los grupos organizados y la capacidad de gestión como gremio. De esta forma, la organización de las TS, en cuya dirección se han informado avances en el Ecuador, ${ }^{26}$ es una medida necesaria.

Por otra parte, el acceso al documento oficial para llevar a cabo el trabajo sexual está limitado en especial por dos circunstancias: la edad y la imposibilidad de superar las pruebas regulares de ITS. En el primer caso, es evidente que no es suficiente con penalizar el trabajo sexual en menores de edad, dado que éste ocurre de cualquier forma, pero en contextos de mayor vulnerabilidad. Es necesario desarrollar medidas que ofrezcan alternativas a las menores de edad, como programas de microcréditos $u$ otro tipo de intervenciones dirigidas a posibilitar una fuente de ingresos alternativa.

Por lo que se refiere a las pruebas de ITS, retirar de forma temporal el documento obliga a pasar a la clandestinidad a las TS que tienen en esta actividad su forma de sustento, lo que también afecta su capacidad de negociación para usar el condón. En este sentido, sería deseable que más que negar el permiso, se educara y promocionaran las medidas de prevención y algún tipo de medios de sustento equivalentes a un seguro de incapacidad temporal.

De modo adicional, es también importante señalar el efecto negativo del precio del preservativo, lo que sugiere que en el Ecuador es deseable incrementar la provisión gratuita de estos dispositivos.

Mención aparte merece el bajo uso del condón con las parejas regulares. Si bien en un contexto de confianza mutua podría no ser un punto esencial de atención, lo cierto es que en el caso de múltiples parejas sexuales e incorrecto empleo del condón con los clientes, es probable que la transmisión de las infecciones a partir de los clientes o las parejas regulares sea más fácil. No obstante, en este caso debe señalarse que las intervenciones factibles necesariamente incluyen a las parejas regulares de las trabajadoras sexuales. 
De igual manera, es importante señalar que este estudio tiene limitaciones en términos de la representatividad de la muestra. Si bien se llevó a cabo un mapeo previo con el fin de construir el marco de muestreo, las TS que participaron son las que resultaron visibles para los entrevistadores que realizaron el mapeo y la encuesta. No obstante, se buscó minimizar este sesgo al incluir a las TS no sólo en el mapeo sino también como entrevistadoras en el equipo de campo de la encuesta.

Asimismo, es importante revisar los resultados obtenidos con el indicador de capital social, que parece sugerir un nexo negativo con el uso de condón. Es preciso llevar a cabo un análisis más detallado de esta variable.

Los resultados notificados en este artículo, la primera muestra grande de TS en el Ecuador, indican que esta población enfrenta un riesgo elevado para contraer ITS. La utilización del preservativo dista de ser consistente y hay grupos específicos en los que se ha incrementado la vulnerabilidad incluso por factores que pueden ser controlables. Se trata de una llamada para intervenciones efectivas en el corto plazo.

\section{Referencias}

1. Bautista CT, Sanchez JL, Montano SM, et al. Seroprevalence of and risk factors for HIV-1 infection among South A merican men who have sex with men. Sex Transm Infect 2004;80(6):498-504.

2. Hierholzer J, Montano S, Hoelscher M, et al. Molecular epidemiology of HIV type 1 in Ecuador, Peru, Bolivia, U ruguay, and Argentina. AIDS Res Hum Retroviruses 2002;18(18):1339-50.

3. Caceres CF. HIV among gay and other men who have sex with men in Latin A merica and the Caribbean: a hidden epidemic? AID S 2002;16 (suppl 3):S23-S33.

4. Schwartländer B, C outinho R, Loures L.The HIV/AIDS epidemic in the Latin America and Caribbean Region. AID S 2002;16(suppl 3):S1-S2. 5. McKeganey N P. Prostitution and HIV: what do we know and where might research be targeted in the future?.AIDS 1994;8(9):1215-26. 6. PubMed [sitio de internet]. N ational Library of Medicine. [C onsultado 2005 mar 21]. Disponible en: http://www.ncbi.nlm.nih.gov/entrez/ query.fcgi.

7.AEG IS Search [sitio de internet]. Aids Education Global Information System. [Consultado 2005 mar 20]. Disponible en: http://

www.aegis.com/search

8. Ringenberg R, Merino ML, Leoro G, et al. HIV infection in Ecuador. Int Conf AID S 1989;5:975 (abstract M.G.0.28). Unique Identifier:AID SLIN E ICA5/00513589.
9. Leoro-Monroy G, Merino ML, Sanchez M, et al.Anti-HIV 1 testing in blood banks in Ecuador. Int Conf AIDS 19894-9;5:1001 (abstract M.G.P.19). Unique Identifier :AID SLIN E IC A5/00527389.

10. Cevallos $R$, D enis F,Verdier $M$, et al. A survey for antibodies to human retrovirus (HIV-1, HIV-2, HTLV-1) in Ecuador. Int Conf AIDS 1990;20-23;6:228 (abstract F.C.588).

11.0 swaldo $R$, Leoro $G$, A guilar $M$, et al. Sentinel serosurveillance for HIV in STD patients, Q uito, Ecuador, 1991-1992. Int Conf AIDS 1993;611:9:731 (abstract PO -C20-3085).

12. Conde-G onzález CJ, Juarez-Figueroa L, U ribe-Salas F, et al. A nalysis of herpes simplex virus 1 and 2 infection in women with high risk sexual behaviour in Mexico. Int J Epidemiol 1999;28(3):571-6.

13. U ribe-Salas F, C onde-G lez CJ, Juarez-Figueroa L, et al. Sociodemographic characteristics and sex practices related to herpes simplex virus type 2 infection in Mexican and Central American female sex workers. Epidemiol Infect 2003;131(2):859-65.

14. Kapiga S, Sam N e, Shao JF, et al. Herpes simplex virus type 2 infection among bar and hotel workers in northern Tanzania: prevalence and risk factors. Sex Transm D is 2003;30(3):187-92.

15. N avindra EP,W inslow IK, Baum MK, et al. Sexually transmitted infections, drug use, and risky sex among female sex workers in Guyana. Sex Transm Infect 2000;76(4):318.

16. Paris M, Gotuzzo E, Goyzueta G, et al. Prevalence of gonococcal and chlamydial infections in commercial sex workers in a peruvian amazon city. Sex Transm D is 1999;26(2):103-7.

17.W H O -UN AIDS. Initiating second generation HIV surveillance systems: practical guidelines. Geneva:W HO -UN AIDS, 2002. 18. A lary M, Mukenge-T shibaka L, Bernier F, et al. Decline in the prevalence of HIV and sexually transmitted diseases among female sex workers in Cotonou, Benin. AIDS 2002;16:463-70.

19. G hys PD, D iallo MO, Ettiegne-Traore V, et al. Increase in condom use and decline in HIV and sexually transmitted diseases among female sex workers in A bidjan, Cote d'Ivoire, 1991-1998. A IDS 2002;16:251-68. 20. Hanenberg RS, Rojanapithayakorn W, Kunasol P, et al. Impact of Thailand's H IV-control programme as indicated by the decline of sexually transmitted diseases. Lancet 1994;344:243-5.

21. Miguez-Burbano MJ,Angarita I, Shultz JM, et al. HIV-related high risk sexual behaviors and practices among women in Bogota, Colombia. Women Health 2000;30(4):109-19.

22. Samuels F, McPherson S, C hikukwa P, et al. Understanding pathways: exploring quantitative social capital measures and their links to health outcomes. En: XV International AID S C onference, 2004:327-334.

23. Holcomb W, Chaiworapongsa T, Luke DA, et al.An odd measure of risk: use and misuse of the odds ratio. Am J 0 bstet Gynecol 2001;98:685-688.

24. Zhang J,Yu K.W hat's the relative risk? A method of correcting the odds ratio in cohort studies of common outcomes. JAMA 1998;280:1690-1.

25. Fenton L. Preventing HIV/AIDS through poverty reduction: the only sustainable solution? Lancet 2004;364(9440):1186-7.

26. Manzo R. Sex-work and HIV prevention in Ecuador: a holistic approach works! The XIV International AID S Conference, 2002 A bstract no. TuPeF5488. 